ResearchArticle

\title{
Study on Solubilization and Stabilization of Eight Flavonoids by 17 Chinese Herbal Polysaccharides
}

\author{
Xuexiao Cao, Lei Wang, Meiqi Liu, Xiaoliang Ren (D), Tingting Han, and Yanru Deng \\ School of Chinese Materia Medica, Tianjin University of Traditional Chinese Medicine, Tianjin 301617, China \\ Correspondence should be addressed to Xiaoliang Ren; renxiaoliang@tjutcm.edu.cn
}

Received 28 April 2020; Revised 30 July 2020; Accepted 6 August 2020; Published 26 August 2020

Academic Editor: Radhey Srivastava

Copyright ( $\odot 2020$ Xuexiao Cao et al. This is an open access article distributed under the Creative Commons Attribution License, which permits unrestricted use, distribution, and reproduction in any medium, provided the original work is properly cited.

\begin{abstract}
Flavonoids are important active components of traditional Chinese medicines (TCMs) because of their many biological activities. We studied the interaction between 17 polysaccharides and eight flavonoids via high-performance liquid chromatography (HPLC) and the effect of the interactions on the solubility and stability of the flavonoids. The effect of the polysaccharides on the solubility of flavonoids was analyzed by statistical methods and showed significant solubility improvements. The constant temperature acceleration method $\left(90^{\circ} \mathrm{C} / \mathrm{pH} 9\right.$ buffer solution) was used to measure the degradation kinetics and half-life of flavonoids with and without polysaccharides. All the polysaccharides displayed a stabilizing effect on all eight flavonoids. The stabilizing effects varied in the order: quercetin, baicalein $>$ baicalin $>$ galuteolin $>$ daidzin $>$ rutin $>$ luteolin $>$ daidzien. The phasesolubility method was applied to quercetin and baicalein to study the mechanism of action of the polysaccharides. It appeared that the two flavonoids could form 1:1 inclusion complexes with polysaccharides, which may be one of the factors increasing solubility and stability. These findings increase our understanding of the role of endogenous polysaccharides in TCM in improving the stability and bioavailability of bioactive flavonoids.
\end{abstract}

\section{Introduction}

Increasing interest in research on traditional Chinese medicine (TCM) has led to the discovery of biological activity and pharmacological effects attributed to increasing numbers of chemical components of TCMs. Some active ingredients cannot be effectively extracted by traditional processes because of low solubility and poor stability, which can not only affect their pharmacodynamics but can also cause wastage of TCM raw materials [1]. Flavonoids are a large class of compounds, widely occurring in plants and also important active ingredients in TCM. They exhibit many biological activities, such as anticancer, antiaging, hepatoprotective, anti-inflammatory, cardiovascular function enhancement, treating chronic prostatitis, enhancing immunity, and regulating the endocrine system [2-6]. Baicalin and baicalein from Scutellaria baicalensis Georgi (SBG) have antibacterial, anti-inflammatory, antioxidant, and neuroprotective effects [7]. Rutin, hesperidin, and D-catechin reduce capillary fragility and abnormal permeability, which can promote hemostasis in capillary hemorrhage, treat hypertension, and act as an auxiliary drug for arteriosclerosis [8,9]. Many flavonoids, however, have poor solubility and stability and, consequently, poor bioavailability. Studies of the compatibility between combinations of herbs used to prepare TCMs found that interactions between components in the different herbs could modify the solubility and stability of the bioactive components [10]. Therefore, finding a way to increase the solubility and stability of the active components in TCMs should increase their bioavailability and, consequently, the efficacy of the TCMs.

Polysaccharides are polymers containing at least 10 monosaccharide monomers linked by glycosidic bonds. They can have a high molecular weight (MW) and may contain hundreds or even tens of thousands of monosaccharide monomers [11]. Polysaccharides are widely distributed in TCMs, especially in Chinese medicines, with a sweet taste [12]. Polysaccharides from TCMs exhibit many biological activities such as antiaging, anticancer, immune regulation, antiradiation, antihypoglycemic, antioxidative, and antibacterial [13-18]. They can also reduce the toxicity of low MW 
compounds and can act as natural stabilizers, solubilizers, and carriers of other active ingredients [19-21]. Similarly, polysaccharides isolated from TCMs show synergistic pharmacological effects when combined with TCMs and improved the bioavailability of some active components [22, 23].

We report a systematic study of the effects of polysaccharides found naturally in TCMs on the solubility and stability of common flavonoid components of TCMs. Seventeen polysaccharides that are found in TCMs were selected for comparison of their effects on 8 representative flavonoids (Figure 1). We established techniques to measure the effect of all possible combinations of polysaccharide and flavonoid on the water-solubility and stability of the flavonoids. In addition, the mechanism of action of the polysaccharides was investigated. The aim was to gain a greater understanding of TCM formulation, ingredient compatibility, and modulation of pharmacological effects, to support the potential application of polysaccharides in TCM formulation.

\section{Methods}

2.1. Materials and Reagents. A total of 17 polysaccharides from traditional Chinese medicine were collected. Astragalus polysaccharide (HQDT) was from Xi'an Seasons Biotechnology Co., Ltd. (Xi'an, China). Cordyceps polysaccharide (CCDT) and Atractylodes polysaccharide (BZDT) were from Lanzhou Baihe Biotechnology Co., Ltd. (Lanzhou, China). Jujube polysaccharide (DZDT), Lycium barbarum polysaccharide (GQDT), and Betula platyphylla polysaccharide (BHRDT) were from Xi'an Tongze Biological Technology Co., Ltd. (Shaanxi, China). Grifola frondosa polysaccharide (HSHDT) and Phellinus chinensis polysaccharide (SHDT) were from Xi'an Wanfang Biological Technology Co., Ltd (Shaanxi, China). Acanthopanax senticosus polysaccharide (CWJDT) was from Ningbo Jin Ainong Biotechnology Co., Ltd (Zhejiang, China). Poria cocos polysaccharide (FLDT) was from Shaanxi Senfu Natural Products Co., Ltd (Shaanxi, China). Polygonatum polysaccharide (HJDT) was from Xi'an Meichuan Biological Technology Co., Ltd (Shaanxi, China). Codonopsis polysaccharide (DSDT) and Polyporus polysaccharide (ZLDT) were from Xi'an Virgin Biotechnology Co., Ltd (Shaanxi, China). Kelp polysaccharide (HDDT) and dandelion polysaccharide (PGYDT) were from Shaanxi Ciyuan Biotechnology Co., Ltd (Shaanxi, China). Achyranthes polysaccharide (NXDT) and Glycyrrhiza polysaccharide (GCDT) were purchased from Xi'an Qingzhi Biotechnology Co., Ltd (Shaanxi, China). Quercetin (wkq16063005), rutin (wkq16063005), baicalein (wkq16011504), baicalin (wkq16070503), luteolin (wkq16051201), galuteolins (wkq16052202), daidzein (150629), and daidzin (wkq16042704) were from Shanghai Ronghe Pharmaceutical Technology Co., Ltd. HPLC-grade formic acid and methanol were from Sigma-Aldrich (St. Louis, MO, USA). Potassium dihydrogen phosphate, sodium hydroxide, and dipotassium hydrogen phosphate were purchased from Tianjin Fengchuan Chemical Reagent Technology Co., Ltd (Tianjin, China).
2.2. Instruments. The water used as the chromatographic mobile phase was purified with a Milli-Q System (Millipore, Bedford, MA, USA). A DELTA 320 pH meter (Mettler Toledo, Greifensee, Switzerland) was used for $\mathrm{pH}$ measurements. A BT125D Lac part analytical balance (Sartorius Scientific Electronic Balance Instrument Co. Ltd, Germany) was adopted for precision measurements. A TG16-WS centrifuge (Changsha Xiangyi Centrifuge Instrument Co. Ltd, China) was used for sample preparation. A Shimadzu HPLC system (Shimadzu-LC-20-AT, Shimadzu, Kyoto, Japan) was used to determine the chemical compositions of the samples.

2.3. Chromatographic Analysis. Chromatographic separation was performed on a Waters XBridge ${ }^{\circledR}$ Shield RP18 column $(4.6 \times 150 \mathrm{~mm}, 5.0 \mu \mathrm{m}$ particle size; Waters, Milford, MA, USA), operated at $30^{\circ} \mathrm{C}$. The mobile phase was composed of methanol (solvent A) and water containing $0.2 \%$ formic acid (solvent B). Isocratic elution programs, with a mobile phase flow rate of $1 \mathrm{~mL} / \mathrm{min}$, were used. The solvent composition was $60 \% \mathrm{~A}: 40 \% \mathrm{~B}$, for quercetin, baicalein, luteolin, and daidzein; $50 \%$ A : 50\% B, for rutin, baicalein, and galuteolin; 40\% A : 60\% $\mathrm{B}$, for daidzin. The detection wavelength was $254 \mathrm{~nm}$ for daidzein and daidzin and $266 \mathrm{~nm}$ for the other six flavonoids. The injection volume was $5 \mu \mathrm{L}$. All solutions were filtered through a $0.22 \mu \mathrm{m}$ nylon membranes before injection into the HPLC.

2.4. Sample Preparation. An excess quantity of each polysaccharide was added to distilled water $(10 \mathrm{~mL})$ and shaken in a water bath at room temperature for $2 \mathrm{~h}$, then centrifuged at $4000 \mathrm{rpm}$ for 15 minutes. Each supernatant was a saturated solution of one of the polysaccharides.

Standard solutions of quercetin $\left(1.02 \mathrm{mg} \cdot \mathrm{mL}^{-1}\right)$, rutin $\left(1.08 \mathrm{mg} \cdot \mathrm{mL}^{-1}\right)$, baicalein $\left(1.03 \mathrm{mg} \cdot \mathrm{mL}^{-1}\right)$, baicalin $(1.00$ $\left.\mathrm{mg} \cdot \mathrm{mL}^{-1}\right)$, luteolin $\left(1.02 \mathrm{mg} \cdot \mathrm{mL}^{-1}\right)$, galuteolin $\left(1.09 \mathrm{mg} \cdot \mathrm{mL}^{-1}\right)$, daidzein $\left(1.06 \mathrm{mg} \cdot \mathrm{mL}^{-1}\right)$, and daidzin $\left(1.00 \mathrm{mg} \cdot \mathrm{mL}^{-1}\right)$ were prepared by dissolving each in methanol.

2.5. HPLC Assay. The standard curve equations of each compound obtained by HPLC were as follows: quercetin $\left(Y=34224 X+11915,1.02-1020 \mu \mathrm{g} \cdot \mathrm{mL}^{-1}\right)$; rutin $(Y=13910 X+$ $\left.4652.6,0.216-1080 \mu \mathrm{g} \cdot \mathrm{mL}^{-1}\right)$; baicalein $(Y=19521 X-87.206$, $\left.0.206-515 \mu \mathrm{g} \cdot \mathrm{mL}^{-1}\right) ;$ baicalin $\quad(Y=19521 X-87.206, \quad 1-1000$ $\left.\mu \mathrm{g} \cdot \mathrm{mL}^{-1}\right)$; luteolin $\left(Y=36006 X-16654,0.102-102 \mu \mathrm{g} \cdot \mathrm{mL}^{-1}\right)$; galuteolin $\left(Y=4169.2 X+6850.1,10.9-1090 \mu \mathrm{g} \cdot \mathrm{mL}^{-1}\right)$; daidzein $\left(Y=6372.7 X-31499, \quad 10.6-159 \mu \mathrm{g} \cdot \mathrm{mL}^{-1}\right) ; \quad$ daidzin $\quad(Y=$ $\left.3641.6 X-8587.1,10-1000 \mu \mathrm{g} \cdot \mathrm{mL}^{-1}\right)$. The correlation coefficients were all $>0.9990$ and the RSD (relative standard deviation) of the retention time for all components $<2.0 \%$. The RSD for the relative peak area was $<2.1 \%$, indicating good precision and repeatability.

2.6. The Effect of TCM Polysaccharide on Water-Solubility of Flavonoids. Excess quercetin, rutin, baicalein, baicalin, daidzein, daidzin, luteolin, or galuteolin was added to the 


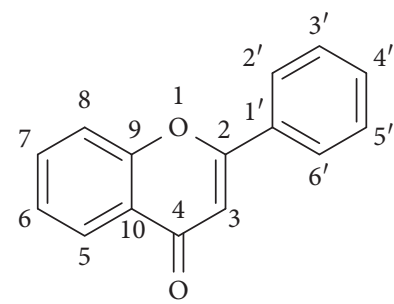

Flavone

Baicalein: 5, 6, 7-OH

Baicalin: 5, 6-OH; 7-glucuronic acid Luteolin: 5, 7, 3', $4^{\prime}-\mathrm{OH}$

Galuteolin: 5, 3', 4'-OH; 7-glucoside

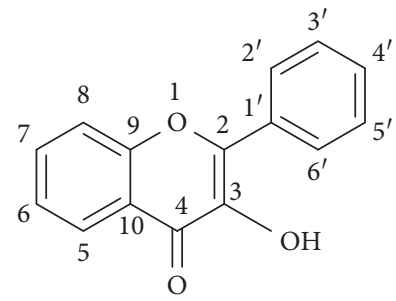

Flavonol

Quercetin: 5, 7, 3', 4'-OH

Rutin: 5, 7, 3', 4'-OH; 3-rutinose

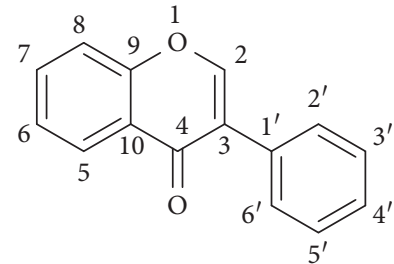

Isoflavone

Daidzein: $7,4^{\prime}-\mathrm{OH}$

Daidzin:4'-OH; 7-glucoside

Figure 1: Chemical structures of 8 flavonoids.

saturated solution of each polysaccharide $(1.0 \mathrm{~mL})$ and placed in a shaking water bath at $45^{\circ} \mathrm{C}$ for 5 hours. After centrifugation at $15,000 \mathrm{rpm}$ for $15 \mathrm{~min}$, the supernatant was diluted with triple volume methanol and stored overnight (about 15 hours) in a refrigerator at $4^{\circ} \mathrm{C}$. The next day, it was centrifuged at room temperature for $15,000 \mathrm{rpm}$ for $15 \mathrm{~min}$. The supernatant was taken as the sample solution and subjected to HPLC analysis.

\subsection{The Effect of TCM Polysaccharide on Stability $\left(\mathrm{pH} 9 / 90^{\circ} \mathrm{C}\right)$} of Flavonoids. Dipotassium hydrogen phosphate $(5.59 \mathrm{~g})$ and potassium dihydrogen phosphate $(0.41 \mathrm{~g})$ were dissolved in water $(1 \mathrm{~L})$ and the $\mathrm{pH}$ adjusted to 9 with sodium hydroxide solution.

The polysaccharide powder was dissolved in 5 times $\mathrm{pH}$ 9 buffer solution and kept slightly boiling for $5 \mathrm{~min}$. The supernatant was centrifuged and reserved. Each flavonoid was weighed into a brown volumetric flask and made up to the mark, by adding one of the above-mentioned polysaccharide supernatants. After $2 \mathrm{~h}$ of shaking in the water bath at $45^{\circ} \mathrm{C}$, the sample was centrifuged for $15 \mathrm{~min}$ at $13,000 \mathrm{rpm}$. The resulting supernatant was a polysaccharide solution of each flavonoid component.

The water solutions and polysaccharide solutions of the flavonoids were sealed, protected from light, and maintained at $90^{\circ} \mathrm{C}$. Samples $(0.5 \mathrm{~mL})$ were removed at specified intervals, diluted with triple volume methanol and centrifuged, and then the supernatant was analyzed by HPLC. The degradation rate constant (Kwater, Kpolysaccharides) ( $k_{\mathrm{W}}$, $k_{\mathrm{P}}$ ) of each flavonoid, in the aqueous and polysaccharide solutions, was calculated using equation (1) and its half-life $\left(t_{1 / 2} \mathrm{~W}, t_{1 / 2 \mathrm{P}}\right)$ was calculated, using equation (2).

$$
\begin{aligned}
\operatorname{Ln}\left(\frac{C_{t}}{C_{0}}\right) & =-k t, \\
t_{1 / 2} & =\frac{\operatorname{Ln}_{2}}{k},
\end{aligned}
$$

where $C_{0}\left(\mu \mathrm{g} \cdot \mathrm{mL}^{-1}\right)$ is the initial concentration in each sample and $C_{t}\left(\mu \mathrm{g} \mathrm{mL}^{-1}\right)$ is the residual concentration at different times.
2.8. Functional Mechanism of Polysaccharides. Excess quercetin or baicalein was added to a saturated solution of each polysaccharide in a brown volumetric flask. After $24 \mathrm{~h}$ in a shaking water bath at $25^{\circ} \mathrm{C}$, a supernatant, saturated with one of the flavonoids, was obtained after centrifuging for $15 \mathrm{~min}$ at $13,000 \mathrm{rpm}$. After dilution with methanol, the concentration of flavonoids was determined by HPLC.

\section{Results and Discussion}

3.1. The Effect of TCM Polysaccharides on the Water-Solubility of Flavonoids. SPSS19.0 software (IBM, Armonk, NY, USA) was used to analyze the influence of polysaccharides on the water-solubility of flavonoids. The data are expressed as mean \pm standard deviation $(x \pm s)$. The $t$-test was used to compare the solubilities in water and polysaccharide solution and determine statistical significance $(P<0.05)$. The saturated solutions of polysaccharides had a significant effect on the water-solubility of all eight flavonoids and generally increased their solubility (Table 1).

The peak area resulting from each flavonoid without polysaccharide was normalized to 1 , and the relative peak area of each flavonoid with polysaccharide was scored. The scoring results were plotted as a heat map (Figure 2). The higher the score, the better the solubilization capacity of the polysaccharide. The solubilities of quercetin, baicalin, daidzein, and luteolin showed the greatest increases, with enhancements up to 68 -fold (for quercetin with CCDT). The GCDT, DSDT, ZLDT, CCDT, HSHDT, NXDT, GQDT, and SHDT had stronger effects in increasing solubility.

The relationship between the solubilization capacity of the polysaccharide and the water-solubility of each flavonoid was plotted for NXDT, CCDT, and GQDT. The watersolubility of each flavonoid was plotted as the abscissa, and the solubilization multiple of each polysaccharide was plotted as the ordinate (Figure 3). The solubilization multiple of each polysaccharide was inversely correlated with the water-solubility of the flavonoids; in other words, the polysaccharides had the greatest solubilization effect on the least soluble flavonoids. It may be related to the equilibrium solubility and structures of flavonoids. Compared with flavonoid glycosides, flavonoid aglycone compounds have more hydroxyl groups and may be more likely to interact 


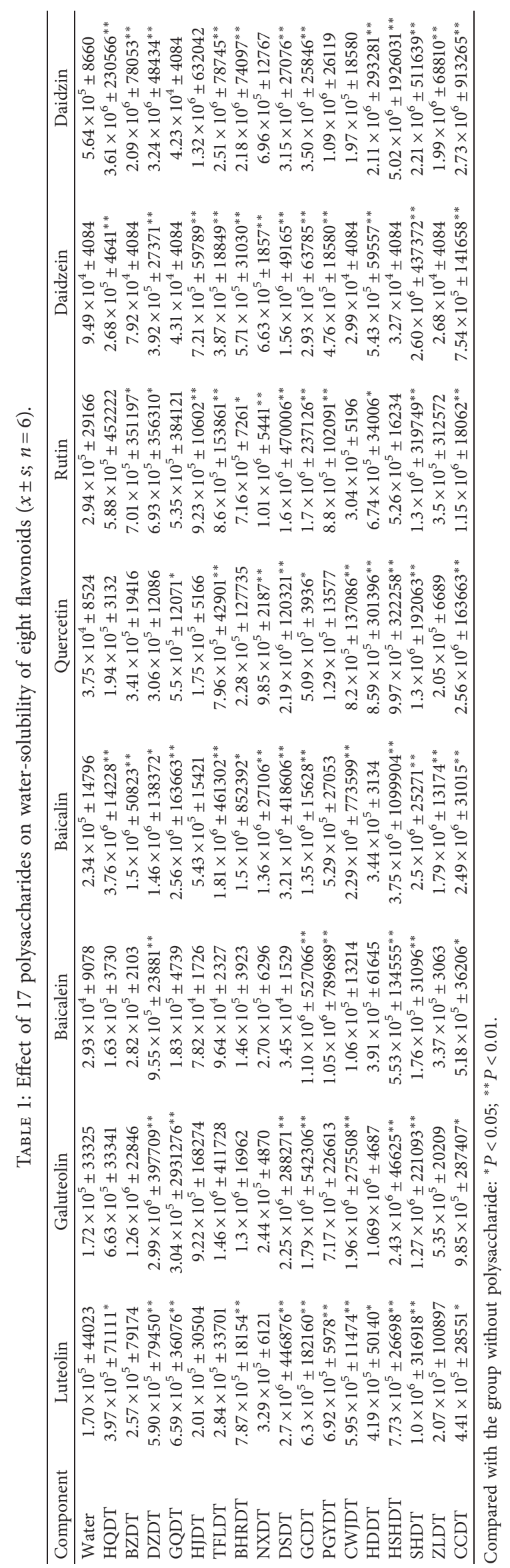




\begin{tabular}{lccccccccc} 
Compound & Luteolin & Galuteolin & Baicalein & Baicalin & Quercetin & Rutin & Daidzein & Daidzin \\
HQDT & 2.34 & 3.85 & 5.56 & 16.07 & 5.17 & 2 & 2.82 & 6.4 \\
BZDT & 1.51 & 7.33 & 9.62 & 6.41 & 9.09 & 2.38 & 0.83 & 3.7 \\
DZDT & 3.47 & 17.4 & 32.6 & 6.24 & 8.16 & 2.36 & 4.13 & 5.745 \\
GQDT & 3.87 & 1.77 & 6.25 & 10.94 & 14.67 & 1.82 & 0.45 & 1.52 \\
HJDT & 1.18 & 5.36 & 2.67 & 2.32 & 4.67 & 3.14 & 7.6 & 2.34 \\
TFLDT & 1.67 & 8.49 & 3.28 & 7.74 & 21.23 & 2.93 & 4.08 & 4.45 \\
BHRDT & 4.63 & 7.56 & 4.98 & 6.41 & 6.08 & 2.44 & 6.02 & 3.87 \\
NXDT & 1.94 & 1.42 & 9.22 & 5.81 & 26.27 & 3.44 & 6.99 & 1.23 \\
DSDT & 1.62 & 13.1 & 1.18 & 13.72 & 58.4 & 5.58 & 16.44 & 5.59 \\
GCDT & 3.76 & 10.4 & 37.54 & 5.77 & 13.57 & 5.85 & 3.09 & 6.21 \\
PGYDT & 4.07 & 4.17 & 35.8 & 2.26 & 3.44 & 3 & 5.02 & 1.92 \\
CWJDT & 3.5 & 11.4 & 3.62 & 9.79 & 21.87 & 1.03 & 0.31 & 0.35 \\
HDDT & 2.46 & 6.2 & 13.34 & 1.47 & 22.9 & 2.29 & 5.72 & 3.74 \\
HSHDT & 4.55 & 14.1 & 18.87 & 16.03 & 26.59 & 1.79 & 0.34 & 8.9 \\
SHDT & 6.18 & 7.38 & 6 & 10.68 & 34.67 & 4.63 & 27.4 & 3.92 \\
ZLDT & 1.22 & 3.11 & 11.5 & 7.65 & 5.47 & 1.19 & 0.28 & 3.53 \\
CCDT & 2.59 & 5.73 & 17.68 & 10.64 & 68.27 & 3.91 & 7.95 & 4.84 \\
\hline
\end{tabular}

FIGURE 2: Heat map showing the effect of polysaccharides on the solubility of flavonoids. The ratio of the solubility with each polysaccharide present to the water-solubility of the flavonoid is shown.

with polysaccharides, thus exhibiting a stronger solubilization and stabilization effect [24]. Follow-up studies were conducted with baicalin and baicalein as examples to preliminary explain the functional mechanism of polysaccharides on flavonoids.

\subsection{The Effect of TCM Polysaccharide on Stability $\left(\mathrm{pH} 9 / 90^{\circ} \mathrm{C}\right)$} of Flavonoids. There was a good linear relationship $\left(R^{2}>0.9025\right)$ between $\operatorname{Ln}\left(C_{t} / C_{0}\right)$ and time for all eight flavonoids in the presence of polysaccharides, indicating that they all exhibited first-order degradation kinetics under the experimental conditions (Table 2) [24]. Quercetin and baicalein, in the absence of polysaccharide, were degraded completely within $10 \mathrm{~min}$ at $\mathrm{pH} 9 / 90^{\circ} \mathrm{C}$. Therefore, no degradation curve was obtained. The half-lives of the leaststable flavonoids (quercetin, baicalein, and baicalin) were extended by some of the polysaccharides from minutes, up to tens or even hundreds of hours (Table 3). Daidzein was relatively very stable in the absence of polysaccharide, and its half-life of $26 \mathrm{~h}$ could only be extended around three-fold at most.

To further visualize the effect of the polysaccharides on the stability of the flavonoids, the ratio of $K_{\mathrm{w}}$ to $K_{\mathrm{ps}}$ was plotted in a heat map (Figure 4). Since $K_{\mathrm{w}}$ could not be measured for quercetin and baicalein, their ratios were infinite, but baicalin showed ratios between 8.5 and 516.5 . $K_{\mathrm{w}}$ for daidzein was much lower than that of the other flavonoids (Table 2), and the polysaccharides had little or no stabilizing effect on it; some even slightly destabilized it. By consulting the literature [25], it was found that daidzein was unstable under alkaline conditions, and its equilibrium solubility in phosphate buffer solution increased with the increase of $\mathrm{pH}$. Polysaccharides had a good stabilizing effect on other flavonoids except for daidzein. It was speculated that there was some interaction between polysaccharides and flavonoids. At the same time, the polysaccharide solution was acidic, which could reduce the $\mathrm{pH}$ of the experimental conditions to a certain extent, thus promoting the stability of flavonoids [24]. The order of stabilizing effects of polysaccharides on the eight flavonoids was as follows: quercetin, $\quad$ baicalein $>$ baicalin $>$ galuteolin $>$ daidzin $>$ rutin $>$ luteolin $>$ daidzien.

In order to better compare the overall stabilizing effects of the different polysaccharides, the degradation rate constants of the flavonoids in water and each of the polysaccharides were compared by principal component analysis (PCA) (Figure 5). From the eigenvalues obtained, principal components were selected, and the cumulative contribution rate $R^{2} X$ (cum) was 0.718 , which indicated that the model was reasonable and had a good analytical ability. All the polysaccharides had a marked stabilizing effect, and the best four overall were HQDT, HSHDT, BHRDT, and CCDT.

\subsection{Functional Mechanism of Polysaccharides on Quercetin} and Baicalein. The relative molecular mass of each polysaccharide was obtained from published values [26-32], and the concentration of each polysaccharide solution was calculated. A phase-solubility diagram was drawn with the polysaccharide concentration $\left(\mathrm{mol} \cdot \mathrm{L}^{-1}\right)$ as the abscissa, and the concentration of quercetin or baicalein $\left(\mathrm{mol} \cdot \mathrm{L}^{-1}\right)$ as the ordinate and a linear relationship was found. According to the Higuchi and Conners methods (Formula (3)): the stability constants $(K)$ were calculated as follows from the slope of the straight line portion of the phase-solubility diagram [33]:

$$
K=\frac{\text { Slope }}{\text { Intercept }(1-\text { Slope })}
$$

The equilibrium binding constants $(K)$ of the inclusion complexes were determined by calculating the slope/intercept. Regression equations, correlation coefficients, and binding constants of phase-solubility of the polysaccharides with quercetin and baicalein are shown in Table 4 . The correlation coefficient $R^{2}>0.9409$ indicated that the linear relationship of phase-solubility was good. The binding constants of the inclusion complexes of the 


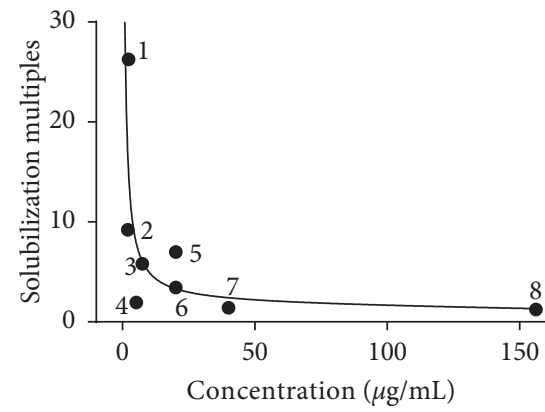

(a)

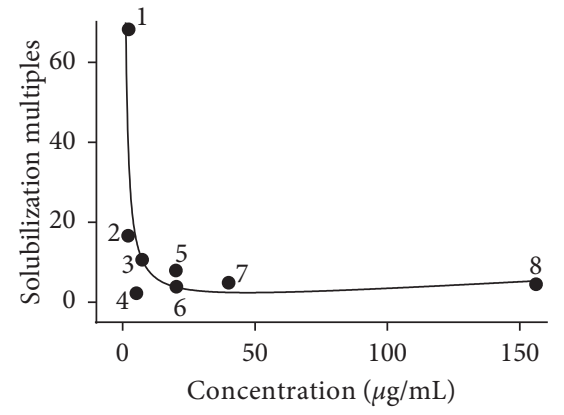

(b)

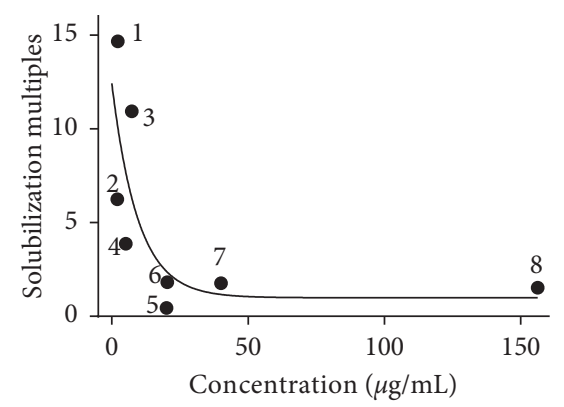

(c)

FIGURE 3: Relationship between solubilization and self-solubility of 8 flavonoids in NXDT, CCDT, GQDT. (a) NXDT; (b) CCDT; (c) GQDT; 1: quercetin; 2: baicalein; 3: baicalin; 4: luteolin; 5: daidzein; 6: rutin; 7: galuteolin; 8: daidzin.

TABle 2: Degradation rate constant $(K)$ of flavonoids at $\mathrm{pH} 9 / 90^{\circ} \mathrm{C}$.

\begin{tabular}{|c|c|c|c|c|c|c|c|c|}
\hline Component & Luteolin & Galuteolin & Baicalein & Baicalin & Quercetin & Rutin & Daidzein & Daidzin \\
\hline$K_{\mathrm{W}}$ & 0.6055 & 0.561 & $\infty$ & 1.8595 & $\infty$ & 0.5496 & 0.0266 & 0.1272 \\
\hline$K_{\mathrm{HQDT}}$ & 0.1224 & 0.0131 & 0.1223 & 0.0113 & 0.1873 & 0.097 & 0.0086 & 0.0055 \\
\hline$K_{\mathrm{HJDT}}$ & 0.2242 & 0.0319 & 0.2546 & 0.0201 & 0.3226 & 0.2105 & 0.0269 & 0.0093 \\
\hline$K_{\mathrm{BZDT}}$ & 0.1854 & 0.0272 & 0.2447 & 0.0129 & 0.2576 & 0.075 & 0.0388 & 0.1107 \\
\hline$K_{\mathrm{HSHDT}}$ & 0.1121 & 0.0158 & 0.1331 & 0.0055 & 0.0966 & 0.059 & 0.0185 & 0.0192 \\
\hline$K_{\mathrm{CWJDT}}$ & 0.1897 & 0.0204 & 0.3383 & 0.0072 & 0.3723 & 0.1711 & 0.0073 & 0.0186 \\
\hline$K_{\mathrm{NXDT}}$ & 0.2068 & 0.0191 & 0.1803 & 0.1569 & 0.4002 & 0.1514 & 0.0372 & 0.0149 \\
\hline$K_{\mathrm{DSDT}}$ & 0.0657 & 0.0475 & 0.6524 & 0.2185 & - & 0.0667 & 0.0081 & 0.0364 \\
\hline$K_{\mathrm{PGYDT}}$ & 0.1172 & 0.0313 & 0.0856 & 0.0523 & 0.2172 & 0.1257 & 0.0264 & 0.0143 \\
\hline$K_{\mathrm{DZDT}}$ & 0.1915 & 0.0025 & 0.0513 & 0.0226 & 0.3492 & 0.1982 & 0.0252 & 0.0143 \\
\hline$K_{\text {SHDT }}$ & 0.1541 & - & 0.1032 & 0.024 & 0.2023 & 0.1916 & 0.0575 & 0.0148 \\
\hline$K_{\text {HDDT }}$ & 0.2677 & 0.0142 & 0.6664 & 0.0036 & 0.4532 & 0.2119 & 0.0148 & 0.0033 \\
\hline$K_{\text {ZLDT }}$ & 0.2343 & 0.0091 & 0.2586 & 0.0057 & 0.4319 & 0.169 & 0.0497 & 0.0276 \\
\hline$K_{\mathrm{BHRDT}}$ & 0.153 & 0.0101 & 0.1425 & 0.0102 & 0.1291 & 0.0536 & 0.0031 & 0.0046 \\
\hline$K_{\mathrm{GQDT}}$ & 0.2165 & 0.043 & 0.0549 & 0.0156 & 0.554 & 0.2125 & 0.0168 & 0.0549 \\
\hline$K_{\mathrm{CCDT}}$ & 0.1445 & 0.0155 & 0.0255 & 0.0062 & 0.3128 & 0.0906 & 0.0071 & 0.0108 \\
\hline$K_{\text {TFLDT }}$ & 0.1918 & 0.0493 & 0.1837 & 0.0473 & 0.3541 & 0.1758 & 0.1562 & 0.0196 \\
\hline$K_{\mathrm{GCDT}}$ & 0.14 & 0.1353 & 0.2305 & 0.0129 & 0.2856 & 0.1227 & 0.0117 & 0.064 \\
\hline
\end{tabular}

TABLE 3: Half-life $t_{1 / 2}(\mathrm{~h})$ of flavonoids at $\mathrm{pH} 9 / 90^{\circ} \mathrm{C}$.

\begin{tabular}{|c|c|c|c|c|c|c|c|c|}
\hline Component & Luteolin & Galuteolin & Baicalein & Baicalin & Quercetin & Rutin & Daidzein & Daidzin \\
\hline Water & 1.14 & 1.24 & - & 0.37 & - & 1.26 & 26.06 & 5.45 \\
\hline HQDT & 5.66 & 52.91 & 5.67 & 61.34 & 3.70 & 7.15 & 80.60 & 126.03 \\
\hline HJDT & 3.09 & 21.73 & 2.72 & 34.48 & 2.15 & 3.29 & 25.77 & 74.53 \\
\hline BZDT & 3.74 & 25.48 & 2.83 & 53.73 & 2.69 & 9.24 & 17.86 & 6.26 \\
\hline HSHDT & 6.18 & 43.87 & 5.21 & 126.03 & 7.18 & 11.75 & 37.47 & 36.10 \\
\hline CWJDT & 3.65 & 33.98 & 2.05 & 96.27 & 1.86 & 4.05 & 94.95 & 37.27 \\
\hline NXDT & 3.35 & 36.29 & 3.84 & 4.42 & 1.73 & 4.58 & 18.63 & 46.52 \\
\hline DSDT & 10.55 & 14.59 & 1.06 & 3.17 & - & 10.39 & 85.57 & 19.04 \\
\hline PGYDT & 5.91 & 22.15 & 8.10 & 13.25 & 3.19 & 5.51 & 26.26 & 48.47 \\
\hline DZDT & 3.62 & 277.26 & 13.51 & 30.67 & 1.98 & 3.50 & 27.51 & 48.47 \\
\hline SHDT & 4.50 & - & 6.72 & 28.88 & 3.43 & 3.62 & 12.05 & 46.83 \\
\hline HDDT & 2.59 & 48.81 & 1.04 & 192.54 & 1.53 & 3.27 & 46.83 & 210.04 \\
\hline ZLDT & 2.96 & 76.17 & 2.68 & 121.60 & 1.60 & 4.10 & 13.95 & 25.11 \\
\hline BHRDT & 4.53 & 68.63 & 4.86 & 67.96 & 5.37 & 12.93 & 223.60 & 150.68 \\
\hline GQDT & 4.53 & 16.12 & 12.63 & 44.43 & 1.25 & 3.26 & 41.26 & 12.63 \\
\hline CCDT & 4.80 & 44.72 & 27.18 & 111.80 & 2.22 & 7.65 & 97.63 & 64.18 \\
\hline TFLDT & 3.61 & 14.06 & 3.77 & 14.65 & 1.96 & 3.94 & 4.44 & 35.36 \\
\hline GCDT & 4.95 & 5.12 & 3.01 & 53.73 & 2.43 & 5.65 & 59.24 & 10.83 \\
\hline
\end{tabular}




$\begin{array}{lcccccccc}\text { Compound } & \text { Luteolin } & \text { Galuteolin } & \text { Baicalein } & \text { Baicalin } & \text { Quercetin } & \text { Rutin } & \text { Daidzein } & \text { Daidzin } \\ K_{\mathrm{W}} / K_{\mathrm{HQDT}} & 4.95 & 42.82 & \infty & 164.6 & \infty & 5.66 & 3.1 & 23.1 \\ K_{\mathrm{W}} / K_{\mathrm{HIDT}} & 2.7 & 17.59 & \infty & 92.5 & \infty & 2.6 & 0.99 & 13.7 \\ K_{\mathrm{W}} / K_{\mathrm{BZDT}} & 3.27 & 20.63 & \infty & 144.1 & \infty & 7.328 & 0.69 & 1.15 \\ K_{\mathrm{W}} / K_{\mathrm{HSHDT}} & 5.4 & 35.5 & \infty & 338.1 & \infty & 9.3 & 0.0185 & 6.6 \\ K_{\mathrm{W}} / K_{\mathrm{CWJDT}} & 3.19 & 27.5 & \infty & 258 & \infty & 3.2 & 3.6 & 6.8 \\ K_{\mathrm{W}} / K_{\mathrm{NXDT}} & 2.93 & 29.37 & \infty & 11.9 & \infty & 3.6 & 0.7 & 8.5 \\ K_{\mathrm{W}} / K_{\mathrm{DSDT}} & 9.22 & 11.81 & \infty & 8.5 & \infty & 8.2 & 3.3 & 3.5 \\ K_{\mathrm{W}} / K_{\mathrm{PGYDT}} & 5.17 & 17.92 & \infty & 35.6 & \infty & 4.4 & 1.007 & 8.9 \\ K_{\mathrm{W}} / K_{\mathrm{DZDT}} & 3.16 & 224.4 & \infty & 82.3 & \infty & 2.8 & 1.06 & 8.9 \\ K_{\mathrm{W}} / K_{\mathrm{SHDT}} & 3.93 & 2 & \infty & 77.5 & \infty & 2.9 & 0.46 & 8.6 \\ K_{\mathrm{W}} / K_{\mathrm{HDDT}} & 2.26 & 39.5 & \infty & 516.5 & \infty & 2.6 & 1.8 & 38.5 \\ K_{\mathrm{W}} / K_{\mathrm{ZLDT}} & 2.58 & 61.6 & \infty & 326.2 & \infty & 3.3 & 0.5 & 4.6 \\ K_{\mathrm{W}} / K_{\mathrm{BHRDT}} & 3.96 & 55.5 & \infty & 182.3 & \infty & 10.3 & 8.6 & 27.7 \\ K_{\mathrm{W}} / K_{\mathrm{GQDT}} & 2.8 & 13 & \infty & 119.2 & \infty & 2.6 & 1.6 & 2.3 \\ K_{\mathrm{W}} / K_{\mathrm{CCDT}} & 4.19 & 36.2 & \infty & 299.9 & \infty & 6.1 & 3.7 & 11.8 \\ K_{\mathrm{W}} / K_{\mathrm{TFLDT}} & 3.16 & 11.4 & \infty & 39.3 & \infty & 3.1 & 0.17 & 6.5 \\ K_{\mathrm{W}} / K_{\mathrm{GCDT}} & 4.33 & 4.1 & \infty & 144.1 & \infty & 4.5 & 2.3 & 2\end{array}$

FIGURE 4: Heat map showing the effect of polysaccharides on the stability of flavonoids. The ratio of the degradation rate constants in water to polysaccharide solution is shown.

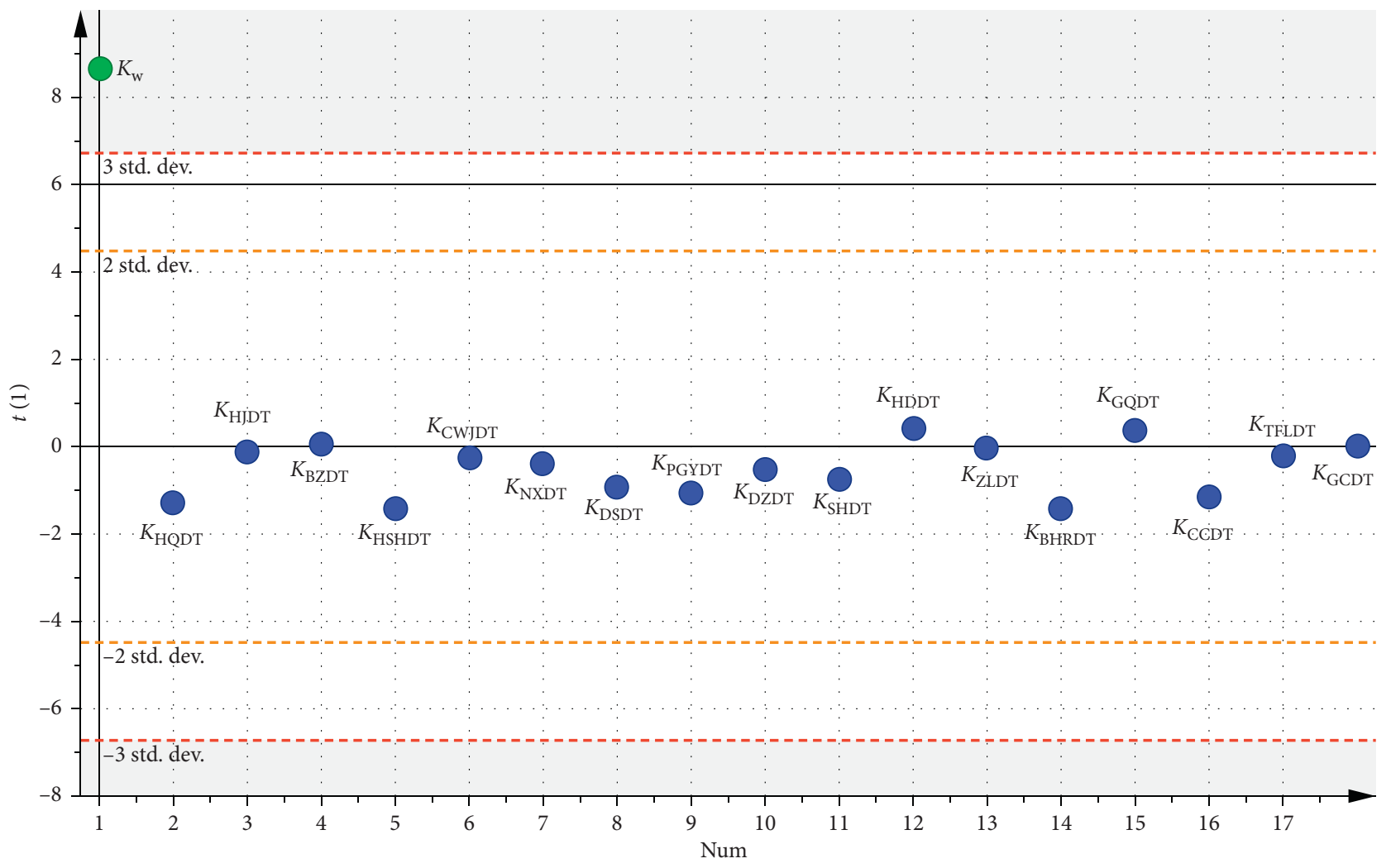

1

FIGURE 5: PCA score plot of degradation constants of flavonoids in water and each of the 17 polysaccharides. 
TABLE 4: Regression equations, correlation coefficients $(r)$, and binding constants of phase-solubility of the polysaccharides with quercetin and baicalein.

\begin{tabular}{lcccccc}
\hline Compound & $\begin{array}{c}\text { Regression equations of } \\
\text { quercetin }\end{array}$ & $R^{2}$ & $\begin{array}{c}\text { Binding constants/ } \\
\text { L.mol }\end{array}$ & $\begin{array}{c}\text { Regression equations of } \\
\text { baicalein }\end{array}$ & $\begin{array}{c}R^{2} \\
\text { Binding constants/ } \\
\text { L.mol }\end{array}$ \\
\hline HQDT & $Y=0.0228 X+5 \times 10^{-7}$ & 0.9860 & 46,664 & $Y=0.0597 X+2 \times 10^{-6}$ & 0.9954 & 31,745 \\
HJDT & $Y=0.0005 X+2 \times 10^{-7}$ & 0.9892 & 2501 & $Y=0.0007 X+2 \times 10^{-6}$ & 0.9954 & 350 \\
BZDT & $Y=0.0007 X+1.6 \times 10^{-6}$ & 0.0032 & 438 & $Y=0.0019 X-9 \times 10^{-7}$ & 0.9974 & 2115 \\
BYRDT & $Y=0.0021 X+3 \times 10^{-7}$ & 0.9886 & 7015 & $Y=0.0026 X+2 \times 10^{-6}$ & 0.9912 & 1303 \\
DSDT & $Y=0.0009 X-2 \times 10^{-6}$ & 0.9936 & 450 & $Y=0.0005 X+2 \times 10^{-6}$ & 0.9890 & 250 \\
GQDT & $Y=0.0013 X+9 \times 10^{-7}$ & 0.9972 & 1446 & $Y=0.0010 X+1 \times 10^{-6}$ & 0.9998 & 1001 \\
NXDT & $Y=0.0023 X+1.5 \times 10^{-6}$ & 0.9876 & 1537 & $Y=0.0032 X+3 \times 10^{-6}$ & 0.9761 & 1070 \\
CCDT & $Y=0.0020 X+2 \times 10^{-7}$ & 0.9974 & 10,020 & $Y=0.0030 X-2 \times 10^{-7}$ & 0.9874 & 15,045 \\
TFLDT & $Y=0.0009 X+1 \times 10^{-6}$ & 0.9900 & 901 & $Y=0.0020 X-3 \times 10^{-8}$ & 0.9950 & 66,800 \\
SHDT & $Y=0.0504 X+6 \times 10^{-7}$ & 0.9958 & 88,458 & $Y=0.0596 X-6 \times 10^{-7}$ & 0.9894 & 105,629 \\
HSHDT & $Y=0.0431 X+4 \times 10^{-7}$ & 0.9781 & 112,603 & $Y=0.0301 X+1.8 \times 10^{-6}$ & 0.9604 & 17,241 \\
CWJDT & $Y=0.0703 X+7 \times 10^{-7}$ & 0.9914 & 108,023 & $Y=0.0524 X-4 \times 10^{-6}$ & 0.9747 & 13,824 \\
ZLDT & $Y=0.0075 X+1 \times 10^{-7}$ & 0.9958 & 75,567 & $Y=0.0550 X+2 \times 10^{-6}$ & 0.9860 & 29,101 \\
DZDT & $Y=0.0260 X-1 \times 10^{-7}$ & 0.9902 & 266,940 & $Y=0.0467 X+2 \times 10^{-6}$ & 0.9908 & 24,494 \\
GCDT & $Y=0.0511 X+1 \times 10^{-6}$ & 0.9884 & 53,852 & $Y=0.2476 X+3 \times 10^{-6}$ & 0.9946 & 109,693 \\
HDDT & $Y=0.0027 X+7 \times 10^{-7}$ & 0.9972 & 3868 & $Y=0.0033 X+2 \times 10^{-6}$ & 0.9948 & 1655 \\
PGYDT & $Y=0.0005 X-2 \times 10^{-8}$ & 0.9769 & 25,013 & $Y=0.0009 X+5 \times 10^{-6}$ & 0.9660 & 180 \\
\hline
\end{tabular}

polysaccharides with quercetin ranged from 438 to $266,940 \mathrm{~L} \cdot \mathrm{mol}^{-1}$ and those of baicalein ranged from 180 to $109,693 \mathrm{~L} \cdot \mathrm{mol}^{-1}$. The phase-solubility diagram of the two flavonoids was a typical AL type, indicating that, within this concentration range, the two flavonoids could form 1 : 1 inclusion complexes with the polysaccharides [34]. These wide ranges suggested that there may be many interactions between polysaccharides, quercetin, and baicalein, such as bonding, encapsulation, and adsorption. The interactions of polysaccharides on flavonoids may be one of the reasons that affect the solubilization and stabilization.

\section{Conclusions}

In this study, the effects of 17 polysaccharides found in

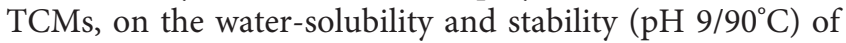
eight flavonoids were investigated. All of the polysaccharides were able to enhance the solubility of all the flavonoids, but the extent of the enhancement varied widely. The greatest effect was observed on the most hydrophobic and least water-soluble flavonoids, quercetin, and baicalein; the smallest effect was observed on the most water-soluble of the flavonoids, luteolin, rutin, and daidzin, which are all glycosides.

The polysaccharides exhibited widely varying stabilization effects on the different flavonoids, essentially, the stabilization was greatest with the least-stable flavonoids (quercetin and baicalein, both of which degraded too rapidly to measure the half-life) and least with the most stable flavonoid (daidzein, which had a half-life of 26 hours under the experimental conditions). The order of stabilizing effects can be summarized as follows: quercetin, baicalein $>$ baicalin $>$ galuteolin $>$ daidzin $>$ rutin $>$ luteolin $>$ daidzein. It is possible that the acidity of some polysaccharide solutions can reduce the $\mathrm{pH}$ of the degradation buffer to a certain extent, thus promoting the stability of the flavonoids.
The mechanism of action of the polysaccharides was studied using the phase-solubility method, applied to quercetin and baicalein, the flavonoids exhibiting the greatest enhancements of solubility and stability. A linear relationship was found between the solubility of quercetin or baicalein and the concentration of the polysaccharide solution. The phase-solubility diagram of quercetin and baicalein was a typical $A_{\mathrm{L}}$ type suggesting that the two flavonoids could form 1:1 inclusion complexes with the polysaccharides. This interaction may be related to the hydrophobicity of the flavonoids because quercetin and baicalein were the most hydrophobic of the flavonoids studied. The inclusion effect of the polysaccharides from TCMs on flavonoids may be one of the factors affecting solubilization and stabilization. Future work should involve the use of Fourier transform infrared (FT-IR), nuclear magnetic resonance (NMR), and other techniques to study the inclusion complexes of polysaccharides and flavonoids and to further explore and verify the mechanism of solubilization and stabilization. The very great increases in solubility and/or stability observed with some of the flavonoid/polysaccharide combinations suggested that there is great potential to modify the formulations of TCMs, particularly by adding exogenous polysaccharides, to achieve marked improvements in both the shelf-life of the medicines and the bioavailability of the active components.

\section{Data Availability}

The data used to support the findings of this study are available from the corresponding author upon request.

\section{Conflicts of Interest}

The authors declare no conflicts of interest. 


\section{Acknowledgments}

This work was financially supported by National Natural Science Foundation of China grants (nos. 81774149 and 81873191).

\section{References}

[1] N. Bou-Chacra, M. Kjc, M. Iac et al., "Evolution of choice of solubility and dissolution media after two decades of biopharmaceutical classification system," The AAPS Journal, vol. 19, no. 4, pp. 1-13, 2017.

[2] Z. F. Wang, J. Liu, Y. A. Yang et al., "A review: the antiinflammatory, anticancer, antibacterial properties of four kinds of licorice flavonoids isolated from licorice," Current Medicinal Chemistry, vol. 27, no. 12, pp. 1997-2011, 2020.

[3] R. V. Patel, B. M. Mistry, S. K. Shinde et al., "Therapeutic potential of quercetin as a cardiovascular agent," European Journal of Medicinal Chemistry, vol. 155, pp. 889-904, 2018.

[4] L. Chen, Y. Wei, S. Zhao et al., "Antitumor and immunomodulatory activities of total flavonoids extract from persimmon leaves in H22 liver tumor-bearing mice," Scientific Reports, vol. 8, no. 1, Article ID 10523, 2018.

[5] X. Y. Liu, L. Xu, Y. Wang et al., "Protective effects of total flavonoids of Astragalus against adjuvant-induced arthritis in rats by regulating OPG/RANKL/NF- $\kappa \mathrm{B}$ pathway," International Immunopharmacology, vol. 44, pp. 105-114, 2017.

[6] K. Sun, L. Xiang, S. Ishihara, A. Matsuura, Y. Sakagami, and J. Qi, "Anti-aging effects of hesperidin on saccharomyces cerevisiaevia inhibition of reactive oxygen species and UTH1 gene expression," Bioscience, Biotechnology, and Biochemistry, vol. 76, no. 4, pp. 640-645, 2012.

[7] X. Liu and C. Liu, "Baicalin ameliorates chronic unpredictable mild stress-induced depressive behavior: involving the inhibition of NLRP3 inflammasome activation in rat prefrontal cortex," International Immunopharmacology, vol. 48, pp. 30-34, 2017.

[8] T. T. Carvalho, S. S. Mizokami, C. R. Ferraz et al., "The granulopoietic cytokine granulocyte colony-stimulating factor (G-CSF) induces pain: analgesia by rutin," Inflammopharmacology, no. 9, pp. 1-12, 2019.

[9] Y. Liu, D. Zhang, Y. Wang et al., "Study on the pharmacokinetics of deoxyschizandrin and schizandrin in combination with epigallocatechin gallate, a component of green tea, in rats," Xenobiotica, vol. 48, no. 4, p. 1, 2018.

[10] S. Wang, Y. Hu, W. Tan et al., "Compatibility art of traditional Chinese medicine: from the perspective of herb pairs," Journal of Ethnopharmacology, vol. 143, no. 2, pp. 412-423, 2012.

[11] X. Cui, S. Wang, H. Cao et al., "A review: the bioactivities and pharmacological applications of Polygonatum sibiricum polysaccharides," Molecules, vol. 23, no. 5, pp. 1170-1180, 2018.

[12] R. Wang, Y. Ji, Y. Peng, and X. Li, "Preliminary characterization of a homogeneous polysaccharide with anticomplement activity from Sijunzi decoction," Journal of Chemistry, vol. 2018, Article ID 8642653, 8 pages, 2018.

[13] C. Y. Shen, J. G. Jiang, L. Yang, D. W. Wang, and W. Zhu, "Anti-ageing active ingredients from herbs and nutraceuticals used in traditional Chinese medicine: pharmacological mechanisms and implications for drug discovery," British Journal of Pharmacology, vol. 174, no. 11, pp. 1395-1425, 2016.

[14] I. A. Schepetkin and M. T. Quinn, "Botanical polysaccharides: macrophage immunomodulation and therapeutic potential,"
International Immunopharmacology, vol. 6, no. 3, pp. 317333, 2006.

[15] L. Zhao, Y. Dong, G. Chen, and Q. Hu, "Extraction, purification, characterization and antitumor activity of polysaccharides from Ganoderma lucidum," Carbohydrate Polymers, vol. 80, no. 3, pp. 783-789, 2010.

[16] N. Li, X. Shen, Y. Liu et al., "Isolation, characterization, and radiation protection of Sipunculus nudus L. polysaccharide," International Journal of Biological Macromolecules, vol. 83, pp. 288-296, 2016.

[17] I. C. F. R. Ferreira, S. A. Heleno, F. S. Reis et al., "Chemical features of Ganoderma polysaccharides with antioxidant, antitumor and antimicrobial activities," Phytochemistry, vol. 114 , pp. $38-55,2015$.

[18] T. Chen, M. Zhang, J. Li, M. M. Surhio, B. Li, and M. Ye, "Structural characterization and hypoglycemic activity of Trichosanthes peel polysaccharide," $L W T$, vol. 70, pp. 55-62, 2016.

[19] W. Cai, M. F. Xia, and Y. K. Xiong, "Study on enhancing efficacy and reducing toxicity of total flavonoids, total polysaccharides in Taxus mairei compatibility with taxol," World Science and Technology/Modernization of Traditional Chinese Medicine and Materia Medica, vol. 17, no. 03, pp. 556-562, 2015.

[20] M. Veverka, T. Dubaj, J. Gallovič et al., "Beta-glucan complexes with selected nutraceuticals: synthesis, characterization, and stability," Journal of Functional Foods, vol. 8, no. 1, pp. 309-318, 2014.

[21] G. J. You, L. L. Sun, X. X. Cao et al., "Comprehensive evaluation of solubilization of flavonoids by various cyclodextrins using high performance liquid chromatography and chemometry," LWT, vol. 94, pp. 172-177, 2018.

[22] D. J. Wang, L. Q. Di, A. Kang et al., "Effect of Bletilla striata polysaccharides on pharmacokinetics of 10 components in Panax notoginseng saponins," Chinese Traditional and Herbal Drugs, vol. 48, no. 4, pp. 737-746, 2017, in Chinese.

[23] H. Bai, Z. Wang, J. Cui et al., "Synergistic radiation protective effect of purified Auricularia auricular-judae polysaccharide (AAP IV) with grape seed procyanidins," Molecules, vol. 19, no. 12, pp. 20675-20694, 2014.

[24] F. Liu, L. L. Sun, G. J. You et al., "Effects of Astragalus polysaccharide on the solubility and stability of 15 flavonoids," International Journal of Biological Macromolecules, vol. 143, pp. 873-880, 2019.

[25] X. Z. Yu, J. He, Y. X. Qian et al., "Determination of equilibrium solubility and apparent oil/water partition coefficient of daidzein by HPLC," Journal of Anhui Agricultural Sciences, vol. 37, no. 10, pp. 4367-4368, 2009, in Chinese.

[26] F. R. Yang, Z. M. Li, and J. P. Gao, "Separation and structural characterization and anti-tumor effect in vitro of polysaccharides from Radix Codonopsis," Lishizhen Medicine and Materia Medica Research, vol. 22, no. 12, pp. 2876-2878, 2011, in Chinese.

[27] S. Li, Y. Zhang, and J. Zhao, "Preparation and suppressive effect of astragalus polysaccharide in glomerulonephritis rats," Advanced Materials Research, vol. 4, no. 1, pp. 554-556, 2007.

[28] S. P. Li, G. H. Zhang, Q. Zeng et al., "Hypoglycemic activity of polysaccharide, with antioxidation, isolated from cultured Cordyceps mycelia," Phytomedicine, vol. 13, no. 6, pp. 428-433, 2006.

[29] J. J. Shan and G. Y. Tian, "Studies on physico-chemical properties and hypoglycemic activity of complex polysaccharide AMP-B from Atractylodes macrocephala Koidz," Acta Pharmaceutica Sinica, vol. 38, no. 6, pp. 438-441, 2003. 
[30] S. Qiu, J. Chen, X. Chen et al., "Optimization of selenylation conditions for lycium barbarum polysaccharide based on antioxidant activity," Carbohydrate Polymers, vol. 103, no. 1, pp. 148-153, 2014.

[31] F. J. Cui, W. Y. Tao, Z. H. Xu et al., "Structural analysis of antitumor heteropolysaccharide GFPS1b from the cultured mycelia of Grifola frondosa GF9801," Journal of Food Science and Biotechnology, vol. 98, no. 2, pp. 395-401, 2006.

[32] Y. Liu, C. Liu, H. Tan, T. Zhao, J. Cao, and F. Wang, "Sulfation of a polysaccharide obtained from Phellinus ribis and potential biological activities of the sulfated derivatives," Carbohydrate Polymers, vol. 77, no. 2, pp. 370-375, 2009.

[33] J. Ji, S. Hao, W. Liu, J. Zhang, D. Wu, and Y. Xu, "Preparation and evaluation of O-carboxymethyl chitosan/cyclodextrin nanoparticles as hydrophobic drug delivery carriers," Polymer Bulletin, vol. 67, no. 7, pp. 1201-1213, 2011.

[34] S. Li, L. Yuan, Y. Chen, W. Zhou, and X. Wang, "Studies on the inclusion complexes of daidzein with $\beta$-cyclodextrin and derivatives," Molecules, vol. 22, no. 12, p. 2183, 2017. 